\title{
Quali le modalità per la nomina a Direttore dell'Osservatorio Astrofisico di Catania? ${ }^{\dagger}$
}

\author{
[1] Socio emerito dell'Accademia Gioenia di Catania
}

Carlo Blanco [1]*

\begin{abstract}
Riassunto
Dall'istituzione dell'Osservatorio Astrofisico di Catania e fino al 1982 la funzione di Direttore era attribuita all'Ordinario di materie astronomiche dell'Università di Catania, per cui le modalità della nomina seguivano i passaggi accademici di assegnazione della Cattedra. Con l'emanazione del D.P.R. 163 del 1982 che istituisce l'autonomia degli Osservatori, la funzione di Direttore, con nomina del Ministro della Pubblica Istruzione a seguito di indicazioni del Consiglio Ricerche Astronomiche, è estesa anche agli Astronomi Ordinari dell'Osservatorio. Con l'istituzione dell'INAF, nel 1999, è il suo Presidente che nomina il Direttore fra quelli selezionati dal Consiglio di Amministrazione con procedure consultive nelle quali è coinvolto anche il personale dell'Osservatorio.
\end{abstract}

Parole chiave: Osservatorio Astrofisico di Catania, Direttore, nomina, astronomi catanesi.

\section{Procedures for the appointment of the Director of Catania Astrophysical Observatory}

\section{Summary}

From the foundation of Catania Astrophysical Observatory and until 1982, the function of Director was assigned to the Full Professor of astronomical subjects of Catania University, therefore the appointment procedure followed the academic process of Chair assignement. Since the D.P.R. 163 in 1982, establishing the Observatory autonomy, the function of Director, with the appointment of the Ministry of Education according to the suggestions of the Astronomical Research Council, has also been extended to the Observatory Senior Astronomers. Since the INAF institution in 1999, the Director has been appointed by the INAF President among those selected by the Administrative Council by means of an advisory procedure involving also the Observatory staff.

Key words: Catania Astrophysical Observatory, Director, appointment procedure, Catania astronomers

\footnotetext{
$\dagger$ Adunanza pubblica, 8 marzo 2019

*e-mail: carloblanco@alice.it
} 
L'emanazione del Decreto 42/2018 dell'Istituto Nazionale di Astrofisica (INAF), contenente le procedure di selezione per la nomina del Direttore dell'Osservatorio Astrofisico di Catania, l'aver spulciato parecchi atti d'archivio per aver pubblicato diversi lavori sulla storia dell' Astronomia catanese e ... per averne vissuti direttamente, mi hanno indotto ad analizzare gli antefatti della nomina di tutti i Direttori dell'Osservatorio catanese. Negli Atti ministeriali non sembra si trovino, per il passato, espliciti bandi selettivi finalizzati alla nomina del Direttore. Nemmeno in quelli universitari, in quanto per un certo periodo di tempo la titolarità direttoriale era conseguente a quella della Cattedra di materie astronomiche dell'Università di Catania. Era infatti il Rettore che, a seguito di indicazioni della Facoltà di Scienze, proponeva al Ministero della Pubblica Istruzione il nominativo per il conferimento della Direzione.

In mancanza di chiare normative mi è sembrato interessante valutare se nella nomina vi fossero, e quali fossero, preferenziali influenze della Scuola astronomica di estrazione dei vari Direttori o dell'Istituzione politica governante nel tempo. L'indagine inizia molto prima dell'istituzione dell'Osservatorio e riguarda l'Astronomia universitaria catanese, sempre concorde a chiederne la costruzione. Vedremo che i meccanismi di nomina non si discosteranno da quelli relativi alla Direzione dell'Osservatorio in senso stretto, almeno fino all'istituzione dell'INAF.

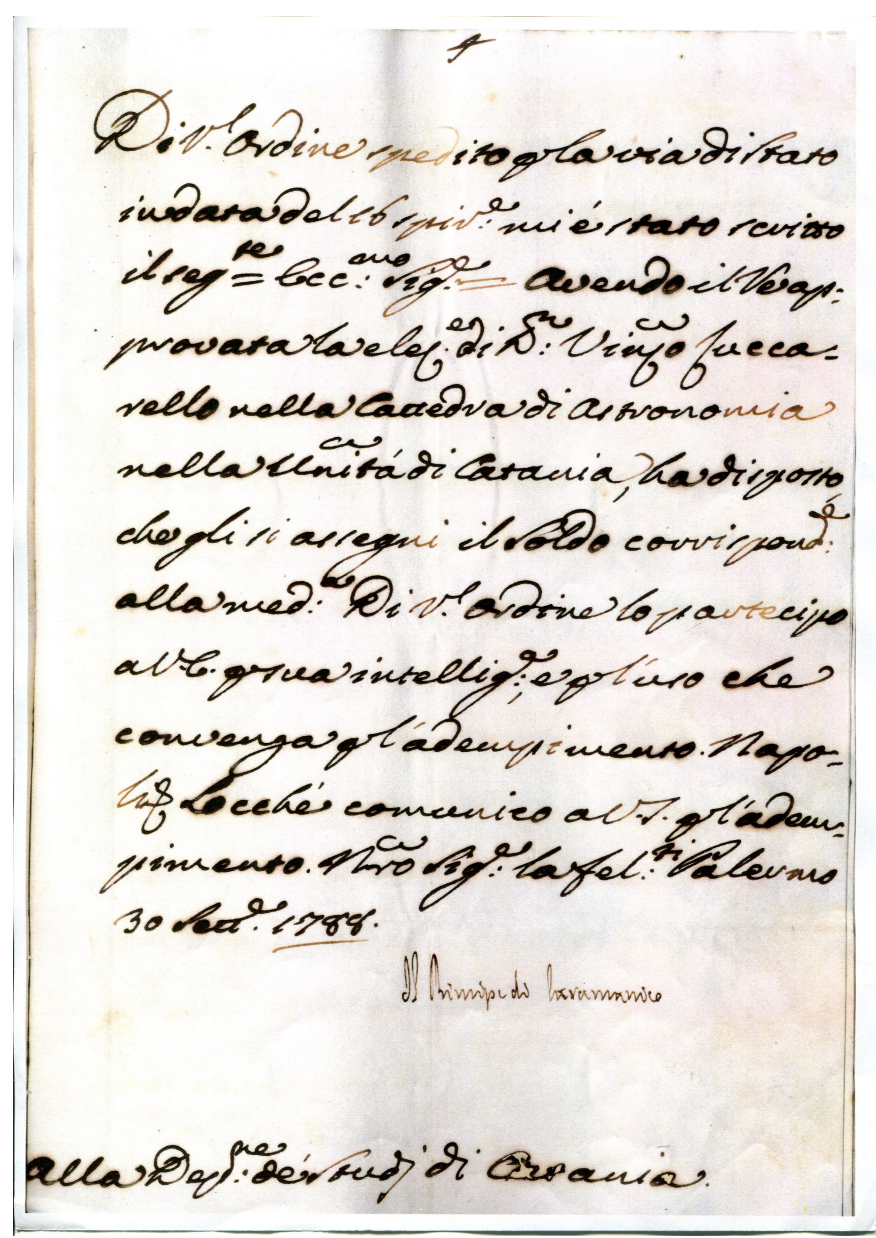

Figura 1 - Biglietto del Vicerè di Sicilia che conferisce l'incarico dell'insegnamento di Astronomia Nautica e Geografia a Vincenzo Zuccarello (1788, ASUCT)

Al 1779 risalgono le prime Istruzioni pelle pubblica generale Università de Studii di Catania [fondata nel 1434] del Vicerè di Sicilia, con le quali viene istituito il Corso analitico [di] Astronomia Nautica e Geografia. Solamente nell'anno accademico 1788/89 il Vicerè di Sicilia, Francesco d'Acquisto, Principe di Caramanico manda il biglietto della nomina al primo docente incaricato Vincenzo Zuccarello. Morto nel 1790, gli succedettero Fortunato Paternò Tedeschi e Giuseppe Orlando. Si erano tutti formati alla scuola matematica catanese ed il loro insegnamento, più che lo studio degli astri, riguardava la meccanica celeste applicata ai sistemi di riferimento (1). 
In questo periodo, dalla lontana Valtellina, arriva a Palermo Giuseppe Piazzi: valente studioso, abile osservatore e preparato insegnante. La sua attività di ricerca imprimerà una svolta a tutta l'Astronomia, non solo siciliana, e renderà l'Osservatorio di Palermo luogo di formazione all'avanguardia. Figlio del celebre Leonardo, filosofo di ispirazione razionalista, Francesco Gambino [o Gambini], fù il primo catanese vero docente di Astronomia. A Palermo per motivi familiari, giovanissimo frequentò il Piazzi formandosi sopratutto nello studio dinamico dei Pianeti. Nel 1794, a soli 18 anni, senza concorso, fù eletto da Ferdinando I alla Cattedra di Astronomia nell'Università di Catania (2). Insegnò per quarant'anni per sovrano comando ... nello studio di Catania ... con soddisfazione ha veduto uscire dalle sue lezioni degl'allievi i quali servono ... o in qualità d'officiali del R. Corpo d'Artiglieria, o in qualità di P. [ubblici] professori di Matematiche (1). Francesco Gambino muore nel 1834 e per otto anni l'insegnamento di Astronomia rimane sospeso. La Deputazione dell'Università di Catania subordina la scelta del nuovo Docente, e di fatto la continuità dell'insegnamento, alla costruzione dell'Osservatorio, continuamente richiesta dal Gambino durante il suo lungo magistero. Il Collegio dei matematici catanesi appronta un piano finanziario per la costruzione che, nel 1837, viene trasmesso alla Commissione assieme alla supplica per avere la cattedra di un giovane matematico, Giuseppe Zurria, caldeggiando un incarico diretto senza concorso. Il Ministro di Stato per gli affari interni in Napoli chiede il parere della Commissione di Pubblica Istruzione di Palermo che si rivolge nuovamente alla Deputazione catanese ed al Direttore dell'Osservatorio di Palermo, Nicolò Cacciatore, i quali esprimono entrambi parere favorevole. In particolare Cacciatore, nella sua relazione manoscritta di ben sette pagine, fà presente che la scelta dei professori mediante concorso moltissime fiate ha fatto preferire il mediocre all'ottimo ... nelle scienze che progrediscono sui fatti è sempre preferibile colui che ha lungo esercizio (1). Il persistente tergiversare nel dare lo sta bene per la costruzione dell'Osservatorio e per la conseguente assegnazione della Cattedra appare come una scelta più politico-economica che scientifico-culturale. Perchè investire sugli studenti e sui professori rivelatisi infidi e rivoluzionari già nel 1820, nel 1827 ed ancor più nel 1848? Proprio in casa di Zurria aveva trovato nascondiglio Salvatore Barbagallo Pittà (direttore del giornale antiborbonico Lo Stesicoreo), uno dei più fervidi cospiratori catanesi. Sorpreso mentre si recava a vedere la moglie ed i quattro figlioletti, fù arrestato e fucilato alla schiena o chianu a statua oggi Piazza dei Martiri. Si andò avanti con incarichi e supplenze affidati per lo più allo stesso Zurria, ormai avviatosi a diventare un brillante matematico.

Non cambiò nulla con l'Unità d'Italia e l'avvento del Regno Sabaudo. Nel 1860 rispondendo ad un'indagine ministeriale sulle cattedre vacanti il Collegio delle Scienze fisiche e matematiche delibera di farsi conoscere al Sig. Rettore ...che vi è propriamente se non una cattedra vuota ed è quella di Astronomia, il di cui insegnamento fù sospeso dal Governo [borbonico] fino a che l'Università avrebbe i mezzi di erigere un osservatorio astronomico ... ora questi mezzi, provenienti dai soldi maturati e non soddisfatti per detta Cattedra, che in somma non indifferente debbono esistere ... e quindi crede di ripristinarsi la detta cattedra di Astronomia, di provvedersi di un professore ... e di darsi cominciamento alla erezione dell'osservatorio (1) (vedi Figura 2). E' noto che la politica piemontese non prevedeva investimenti nell' annesso meridione d'Italia, anzi la storia racconta esattamente il contrario. Il Dicastero della Istruzione Pubblica della Luogotenenza generale del Re nelle province siciliane fa sapere che non ci sono risorse realmente disponibili, quindi niente cattedra nè osservatorio. 


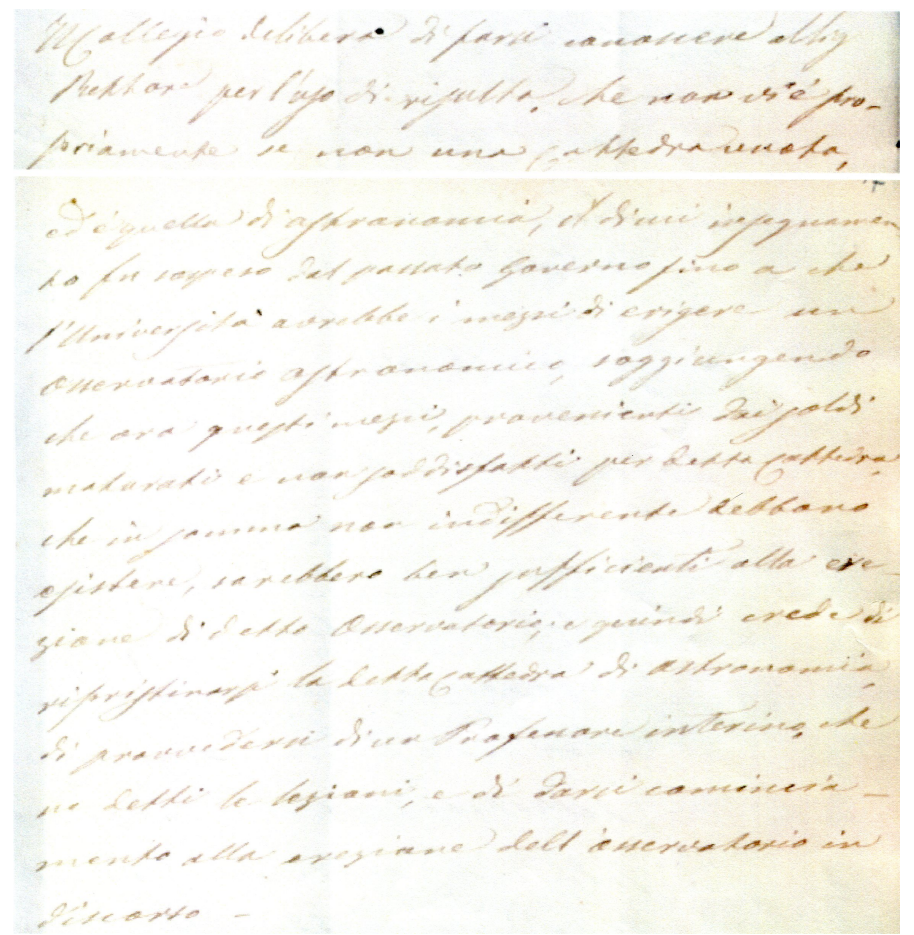

Figura 2 - Delibera del Collegio delle Scienze fisiche e matematiche dell'Università di Catania relativa ad un'inchiesta del Ministero sulle Cattedre vacanti (1860,ASUCT).

Referente del Regno Sabaudo per l'astronomia era Giovanni Schiapparelli, direttore dell'Osservatorio di Milano. Dovendo rilanciare l'Osservatorio di Palermo alla cui direzione era stato reintegrato Nicolò Cacciatore, fiaccato fisicamente per il duro esilio (3), inviò a Palermo come astronomo il giovane Direttore dell'Osservatorio di Modena Pietro Tacchini. Forte di prestigio personale e di importanti relazioni politiche ed accademiche, volendo portare avanti le ricerche astrofisiche che conduceva in collaborazione con Angelo Secchi, Tacchini trovò il modo, sempre in servizio a Palermo, di istituire il Regio Osservatorio Astrofisico di Catania ed attivare la cattedra di Astronomia fisica nella locale Università (4). A presiederli fece venire Annibale Riccò, anche lui modenese ed astronomo all'Osservatorio di Palermo, dando così inizio alla colonizzazione dell'Astronomia catanese. 


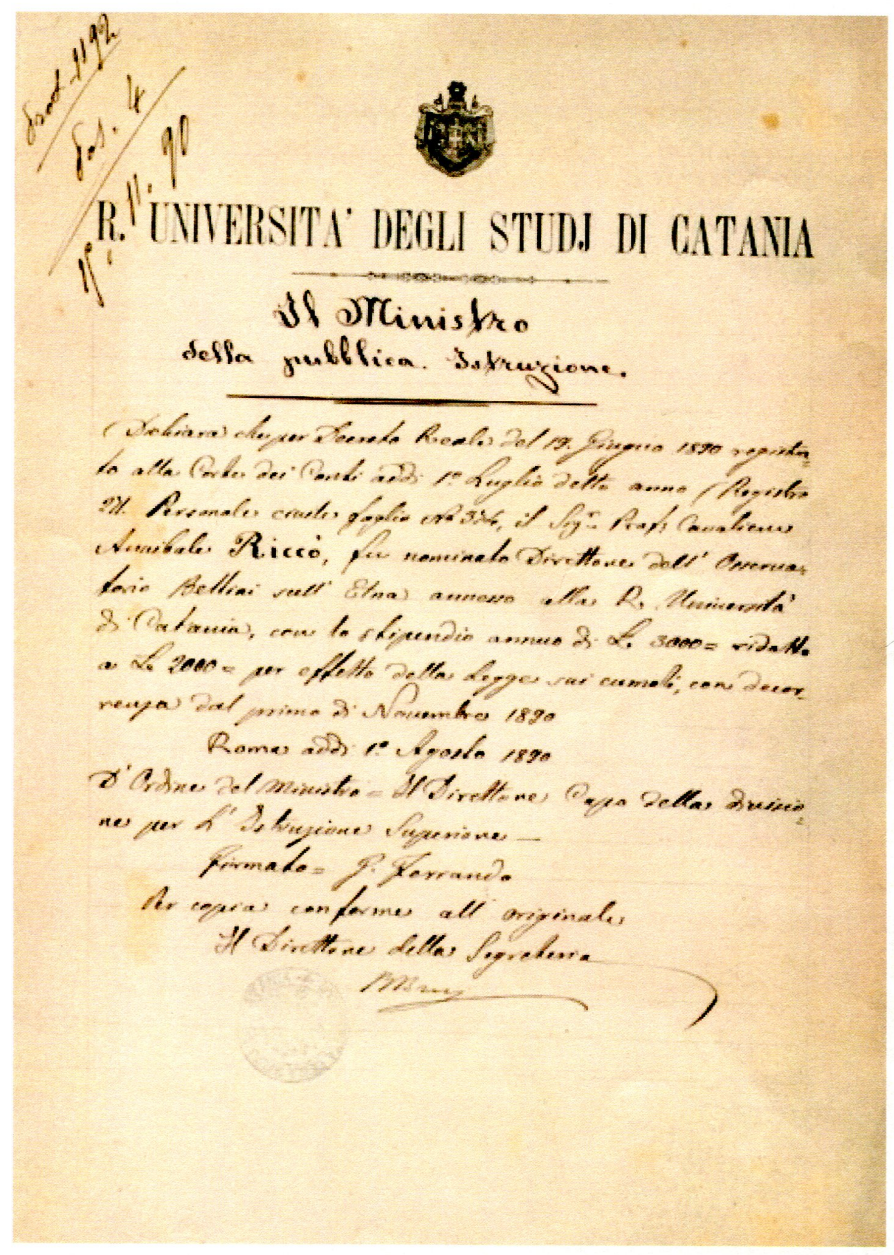

Figura 3 - Decreto ministeriale di nomina di Riccò a Professore Ordinario di Astronomia fisica (1890, ASUCT).

La super attività di Tacchini fece sì che l'Osservatorio catanese fosse prescelto, unico italiano, fra i 18 prestigiosi Osservatori incaricati di compilare la Carte du Ciel (5). L'assegnazione, che nelle idee di Tacchini doveva contribuire a sviluppare la poliedricità degli indirizzi di ricerca del giovane Osservatorio catanese, di fatto ne condizionerà l'attività per più di mezzo secolo, cambiandone l'indirizzo di ricerca prevalente ed addirittura determinando il cambio del nome della cattedra da Astronomia fisica in Astronomia con elementi di Geodesia. L'attività di Riccò realizzò tutti i programmi di ricerca di Tacchini, tranne che per l'Osservatorio in cima all'Etna. D'altronde Tacchini nel proporne la costruzione era stato attratto dal ridotto assorbimento atmosferico ma, da buon modenese, non conosceva le difficili condizioni meteorologiche di un'elevazione di più di 3000 metri di altezza isolata in mezzo al Mediterraneo ed i disturbi arrecati dal vicino cratere centrale attivo. Riccò fu oberato da tanti incarichi da, forse involontariamente, trascurare la formazione di una Scuola catanese o non ne avvertì la necessità avendo avuto, tranne il catanese Mascara, validi collaboratori, quali Bemporad e Favaro, formatisi in altri osservatori con competenze adatte al particolare lavoro della compilazione della Carte. 


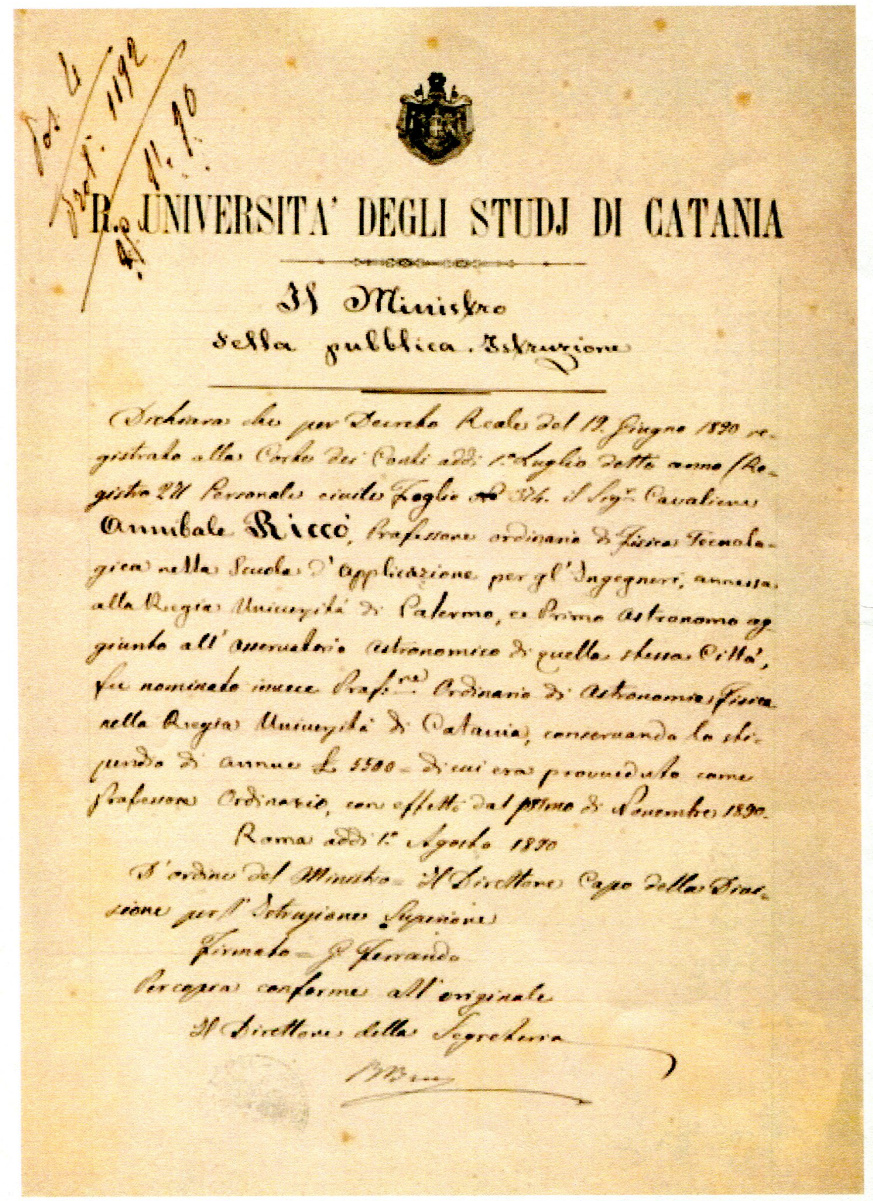

Figura 4 - Decreto ministeriale di nomina di Riccò a Direttore dell'Osservatorio Bellini sull'Etna, successivamente Regio Astrofisico di Catania (1890, ASUCT).

Proprio Favaro, venuto a Catania come astronomo nel 1914, succedette a Riccò, morto di malaria nel 1919 a Roma. Laureatosi a Padova, aveva acquisito una grande pratica nell' astronomia di posizione e dopo la parentesi bellica torna a Catania dove, dal 1919 al 1921, insegna un corso libero, fin quando nel 1922 viene cambiato il nome dell'insegnamento in Astronomia con elementi di Geodesia, che gli viene assegnato come cattedra. Dal 1924 è Direttore dell'Osservatorio fino al 1933 quando si trasferisce a Trieste. Sarà ancora Direttore incaricato a Catania dal 1938 al 1943 durante l'allontanamento di Azeglio Bemporad in conseguenza delle Leggi razziali.

Questi era stato assistente all'Osservatorio Astrofisico di Catania dal 1904, dedicandosi prevalentemente al lavoro della Carte du Ciel fino al 1912 quando diventa Direttore dell'Osservatorio di Napoli Capodimonte, da dove continuò a lavorare per la Carte. Nel 1933, resisi vacanti la Cattedra di Astronomia e la Direzione dell'Osservatorio per il trasferimento di Favaro, disturbato dalle molestie razziali perchè ebreo, venne a Catania, sperando in una minore intensità dei moti antisemitici nella città etnea. Nel 1938 l'emanazione dei Decreti razziali porta al suo esonero fino al 1943. Reintegrato cerca di riavviare l'astronomia catanese ma i patimenti subiti (morte della moglie, distruzione della casa per un bombardamento aereo, privazione del completamento della Carte $\mathrm{du}$ Ciel) avranno contribuito alla morte per infarto nel 1945.

Nell'avvicendarsi delle diverse Direzioni la continuità della vita dell'Osservatorio fù assicurata da Antonino Taffara, dal 1901 calcolatore nei ruoli dell'Osservatorio. Fù lui che nel 1942 in una seduta dell'Accademia Gioenia annunciò il completamento della compilazione della Carte du Ciel. 


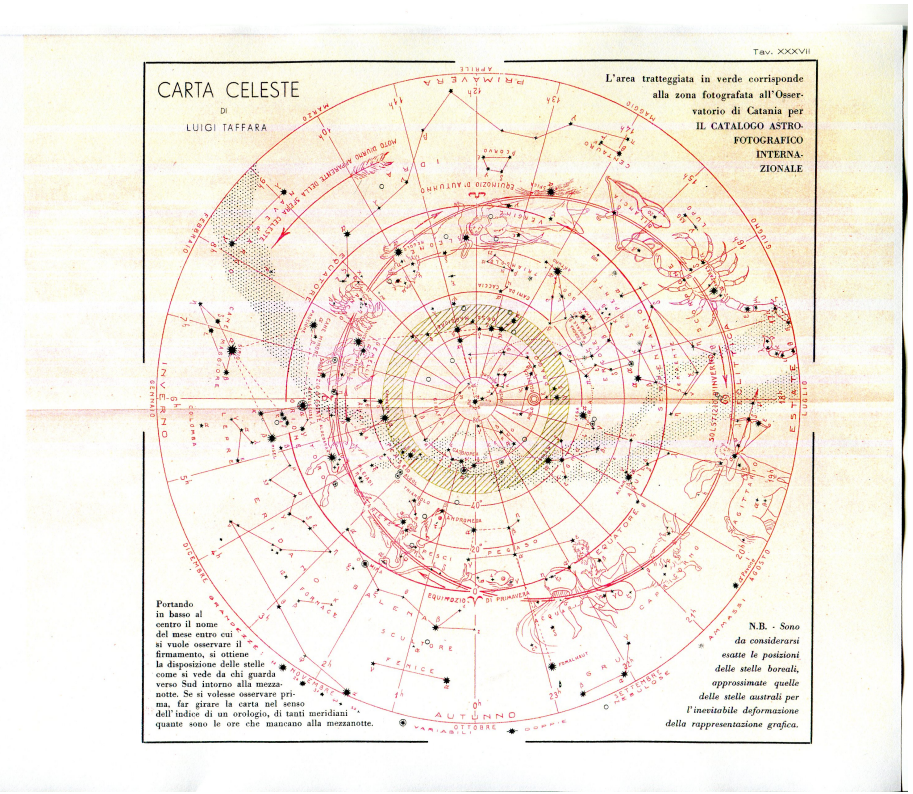

Figura 5 - Carta celeste di Luigi Taffara. L'area tratteggiata corrisponde alla zona fotografata all'Osservatorio Astrofisico di Catania per la Carte du Ciel. "Il Regio Osservatorio Astrofisico di Catania all'Esposizione Universale di Roma (1942)", Tav. XXXVII.

Laureatosi in Ingegneria civile a Napoli, dopo aver frequentato i corsi di Astronomia e Geodesia, Eugenio De Caro entra nei ruoli degli Osservatori nel 1926. Astronomo aggiunto a Catania dal 1934 al 1937, dopo la parentesi bellica con i gradi di Ufficiale del Genio e la prigionia, nel 1946 ritorna a Catania come titolare del corso di Astronomia e Geodesia e Direttore dell'Osservatorio. La sua attività più che dalla ricerca e dalla didattica fù assorbita dalla difesa dell'esistenza fisica dell'Osservatorio e dall'assicurazione della continuità delle osservazioni solari che, malgrado l'impresa della Carte du Ciel, erano state regolarmente condotte (nel 1925 erano stati registrati 332 su 365 giorni di osservazione) (4). De Caro muore nel 1954 lasciando un Osservatorio mal ridotto, con sostanzialmente le stesse attrezzature, ormai obsolete, dei tempi di Riccò, le strutture soffocate dall'espansione dei padiglioni ospedalieri e della città, ma sopratutto senza una frequentazione interessata di studenti, per lo più del Corso di laurea in Matematica, che sceglievano Astronomia con elementi di Geodesia come materia complementare. Non poteva essere diversamente con Docenti formatisi in altre sedi che svolgevano la loro funzione di didatta e di astronomo con competenza e professionalità ma che sentivano la loro presenza a Catania come una situazione temporanea da interrompere.

Nel più di mezzo secolo di esistenza l'attività dell'Osservatorio aveva evidenziato la bontà del cielo catanese per le osservazioni della fenomenologia solare. Naturale che il fiorentino Giorgio Abetti, Direttore dell'Osservatorio Astrofisico di Arcetri, in quel periodo all'apice delle ricerche solari, ponesse l'attenzione sull'Osservatorio catanese (unico Osservatorio Astrofisico italiano oltre Arcetri). Il suo disegno scientifico lo manifestò nella conferenza inaugurale del CXXXI anno accademico della Gioenia (4), suggerendone come realizzatore Mario Girolamo Fracastoro, suo allievo ad Arcetri, nel 1954 chiamato a Catania come Ordinario e diventato, quindi, Direttore dell'Osservatorio. Nell' anno accademico 1955-56 ripristina il corso di Astronomia indirizzandone i contenuti verso la Fisica. Negli anni immediatamente seguenti si adopera per attivare nel Corso di Laurea in Fisica gli insegnamenti di Astrofisica, Fisica solare, Meccanica celeste, dandoli per supplenza o incarico annuale a brillanti neolaureati in Fisica. Le tesi di laurea erano prevalentemente di carattere osservativo, sia solare che stellare. Queste ultime, dopo un breve avvio a S. Agata Li Battiati, si conducevano presso la nuova Sede Stellare (oggi porta il suo nome) a 1800 metri di quota sull'Etna, realizzata con i proventi della cessione all'Ospedale V. Emanuele del giardino della vecchia Sede di Piazza Vaccarini. Ben presto trovò il modo di trattenere, per lo più per incarico annuale nei ruoli dell'Università o dell'Osservatorio (oggi si chiamerebbero precari), i laureati più idonei a fare ricerca, gettando le fondamenta di una Scuola catanese di astronomi. 
Improvvisamente nel 1965, quando sembrava avesse messo radici nella città etnea, liberatasi la Direzione dell'Osservatorio a Torino, Fracastoro, vi si trasferisce.

Sempre da Firenze nel 1966 arriva Giovanni Godoli. Con l'entusiasmo della prima Cattedra e Direzione, sprona i giovani reclutati da Fracastoro ad affrontare i concorsi nazionali per occupare in ruolo le posizioni tenute come precari. Si forma una Scuola catanese, che si affermerà in campo internazionale con una propria originale linea di ricerca sull'Attività stellare di tipo solare (6), riavvicindosi all'Astrofisica, come d'altronde imponeva il nome dell'Osservatorio.

Sull'onda delle contestazioni del 1968 le Università gradatamente apportano radicali modifiche ai loro Statuti e Regolamenti. Fra le innovazioni l'aumento dei Corsi di Laurea, degli insegnamenti curriculari (sui quali dopo tre anni consecutivi di insegnamento della stessa materia ci si poteva stabilizzare) e delle fasce della Docenza in Ordinari, Associati e Ricercatori. Sono una diecina gli insegnamenti tenuti dagli astronomi catanesi, come secondo incarico, nelle Facoltà di Scienze ed Ingegneria delle Università di Catania e Messina.

Da Padova arriva Paolo Maffei, chiamato sulla nuova Cattedra di Astronomia istituita nel 1973 nel Corso di Laurea in Fisica, ferma restando a Matematica quella alla quale, per il D.P.R. n.6 dell'11 gennaio 1956, è legata la Direzione dell'Osservatorio.

Nell'anno accademico 1976-77 Godoli è idoneo in un concorso a cattedra in Fisica solare a Firenze e ritorna nella sede di provenienza. Nell'insegnamento di Astronomia a Matematica, rimasto vuoto, dapprima come incaricato e dal 1978 come incaricato stabilizzato, gli subentra lo scrivente. La Direzione dell'Osservatorio, quale unico Ordinario di Astronomia, viene assegnata a Paolo Maffei che la tiene fino al 31 ottobre del 1980 quando si trasferisce a Perugia, nella natia Umbria.

Nella riunione del 12 gennaio 1981 il Consiglio della Facoltà di Scienze catanese, preso atto della rinuncia del Prof. Claudio Chiuderi, anche lui fiorentino, a ricoprire la Cattedra di Astronomia messa a concorso perchè chiamato ad altra cattedra presso l'Università di Firenze, mi indica al Ministero della Pubblica Istruzione come Direttore dell'Osservatorio di Catania, in quanto Stabilizzato sull'insegnamento di Astronomia del Corso di Laurea in Matematica cui è legata la Direzione dell'Osservatorio. Il Ministro, con lettera del 18 luglio 1981, mi conferisce, con effetto immediato e nelle more della nomina del titolare della cattedra di Astronomia, la direzione dell'Osservatorio Astrofisico di Catania. Dopo quasi un secolo di esistenza dell'Osservatorio, la direzione è conferita ad un catanese, ponendo fine alla colonizzazione iniziata con Riccò. 


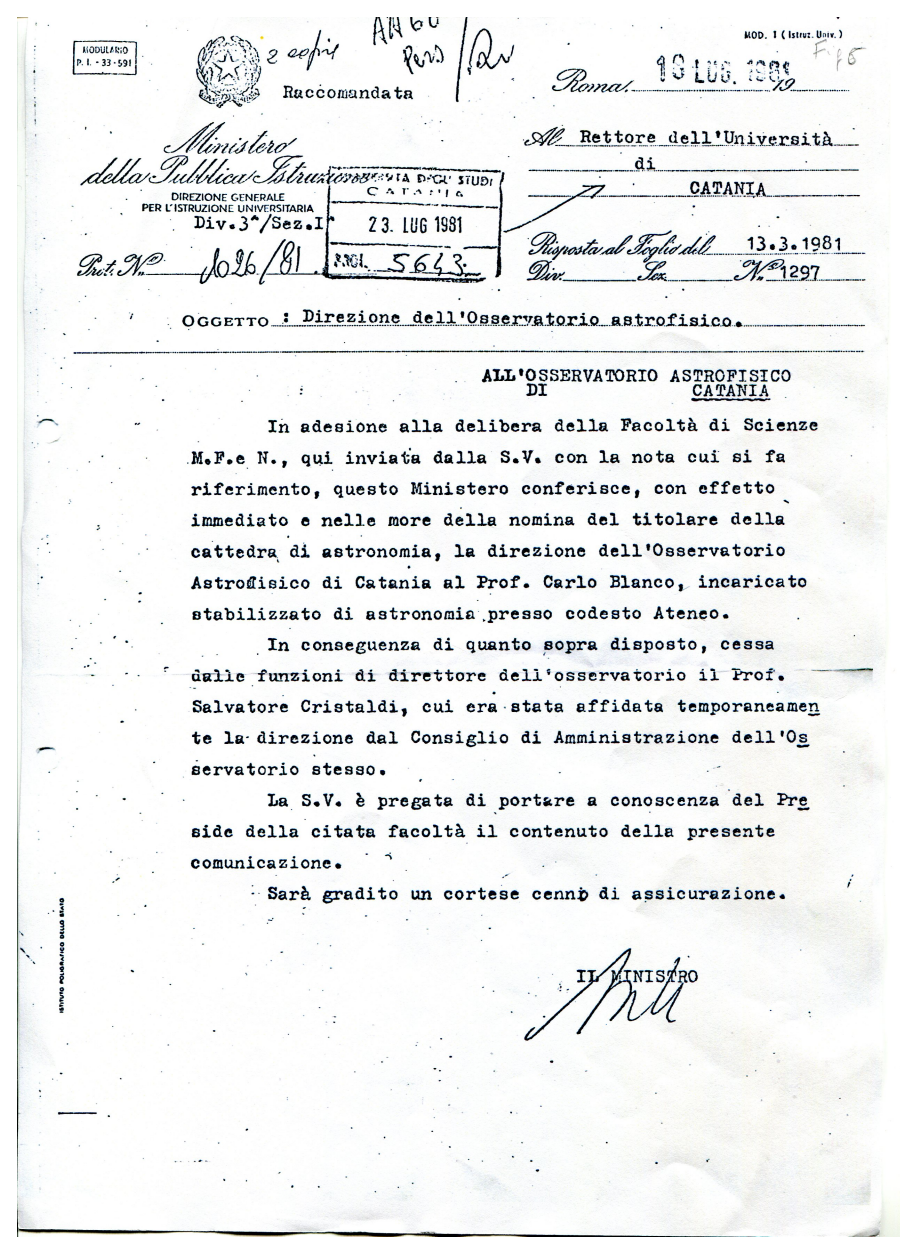

Figura 6 - Decreto del Ministro della Pubblica Istruzione che conferisce a Carlo Blanco la nomina a Direttore dell'Osservatorio Astrofisico di Catania.

Nel 1982 alla prima tornata dei Giudizi di idoneità a Professore associato nel Raggruppamento disciplinare Astronomia risultano idonei ben sette catanesi, sancendo anche la loro validità di docenti.

Il vento innovativo post '68 soffia anche sugli Osservatori. Con riunioni a carattere nazionale si sviluppa un lungo dibattito sui rapporti Osservatori-Università, fra chi propone il loro inglobamento come Dipartimenti atipici nelle sedi universitarie viciniori e chi ne chiede il distacco. Altri ritengono che strutture ed organici di alcuni Osservatori siano troppo piccoli e propongono la costituzione di un unico Osservatorio nazionale, vedendo così facilitata la realizzazione del progetto del Telescopio nazionale di 5 metri di diametro. Nel 1982 viene emanato il D.P.R. 163 che stabilisce l'autonomia degli Osservatori e ne adegua la struttura amministrativa. Tra le novità che interessano questa rassegna: la suddivisione del personale scientifico in tre fasce analoghe a quelle universitarie (astronomi ordinari, astronomi associati e ricercatori astronomi) e la Direzione degli Osservatori non più legata esclusivamente alla Cattedra di Astronomia ma assegnata ad un Professore ordinario o straordinario di discipline astronomiche o agli Astronomi ordinari o straordinari degli Osservatori. Le nomine, con durata di un trennio e rinnovabili solamente per un altro triennio, vengono fatte dal Ministro della Pubblica Istruzione a seguito di proposta del Consiglio Ricerche Astronomiche (CRA), anch'esso istituito con il D.P.R. 163/82. Presieduto dal Ministro della Pubblica Istruzione è composto da quattro Professori universitari (almeno due ordinari ed un associato), quattro astronomi (almeno due ordinari ed un associato), un rappresentante dei ricercatori universitari ed uno degli Osservatori, due rappresentanti di Enti pubblici di ricerca operanti in campo astronomico e due rappresentanti ministeriali. Nella prima costituzione la rappresentanza del personale degli Osservatori, non ancora esistente, era sostituita da quattro Direttori di Osservatorio. 
Nella seduta del 14 ottobre 1983 il CRA, ai sensi dell'art. 6 del D.P.R. 163/82, propone il rinnovo del mio mandato a Direttore dell'Osservatorio Astrofisico di Catania a decorrere dall'1 novembre 1983, per la durata di un triennio.

In quell'anno vengono messi a concorso nazionale 10 cattedre di Astronomia e per la prima volta 10 posti di Astronomo ordinario. Fra gli universitari risulta idoneo Marcello Rodonò, primo Ordinario catanese in materie astronomiche dopo Francesco Gambino. Rodonò sarà nominato Direttore dell'Osservatorio dal 1 gennaio 1987 (il mio mandato era stato prolungato di due mesi per completare l'anno solare) e dirigerà l'Osservatorio fino al 31 dicembre 2001.

Intanto il travaglio degli Osservatori continua non essendo il loro assetto giuridico - istituzionale al passo con i tempi. Sono cominciate le osservazioni dallo spazio e sulla Terra entrano in funzione e sono in cantiere telescopi di sempre maggiori dimensioni in siti posti in alte montagne in località isolate, maggiormente nell'emisfero Sud. Queste imprese molto costose sono gestite da cooperazioni internazionali (ESO, ESA, VLBI, LBT, ..) che richiedono un'unica rappresentanza decisionale nazionale, non prevista nella legge istitutiva degli Osservatori nè del CRA. Alle carenze finanziarie si cerca di far fronte con il Consorzio Nazionale per l'Astronomia e l'Astrofisica (CNAA). Con il Decreto Legislativo del 23 luglio 1999, numero 296, nasce l'INAF, Ente di ricerca non strumentale ad ordinamento speciale, con sede in Roma e con strutture operative distribuite sul territorio nazionale, rappresentate dagli Osservatori astronomici ed astrofisici e dal CNAA. Dopo qualche anno vi confluiscono anche gli Istituti del CNR di Radioastronomia, Fisica spaziale e Fisica dello spazio interplanetario.

Marcello Rodonò lascia la direzione dell'Osservatorio per dirigere il Dipartimento strutture dell'INAF. A seguito del bando di selezione pubblica previsto dal Regolamento sull'organizzazione e funzionamento dell'INAF per la nomina dei Direttori dei 12 Osservatori astronomici ed astrofisici e della susseguente valutazione delle domande da un'apposita commissione, con Decreto n. 19 del Presidente dell'INAF, dal primo gennaio 2002 e per un triennio viene nominato Direttore dell'Osservatorio Astrofisico di Catania Santo Catalano. Astronomo ordinario nei ruoli dell' INAF, è il primo direttore dell'Osservatorio catanese non proveniente dai ruoli dell'Università di Catania.

Con Decreto Legislativo 4 giugno 2003, n. 138 viene emanato il riordino dell'INAF. L'art. 13 prevede che i Direttori degli Osservatori siano nominati dal Consiglio di Amministrazione dell' INAF sulla base delle competenze scientifiche e manageriali possedute ed a seguito di procedure selettive definite dai Regolamenti dell'Ente. L'incarico di Direzione è a tempo pieno, dura cinque anni e può essere rinnovato una sola volta.

Terminata alla fine del 2004 la direzione di Santo Catalano, il primo gennaio 2005 torna come Direttore Marcello Rodonò, ma la morte improvvisa il 25 ottobre di quell'anno interrompe il suo mandato. Nelle more dell'espletamento delle procedure selettive per la nomina del nuovo direttore, dall'1 novembre e fino al 28 febbraio 2006, l'Osservatorio è diretto da Santo Catalano.

Dal successivo 1 marzo per un quinquennio, come dettato nel Decreto Legge del Riordino dell'INAF, viene nominato Direttore Gianni Strazzulla, anche lui Astronomo ordinario nei ruoli dell'Osservatorio. E' il primo Direttore proveniente dalla seconda generazione di astronomi formatisi nella scuola catanese. Avendo rinunciato a candidarsi per un secondo mandato, il 31 marzo 2011 la sua direzione termina.

Le susseguenti selezioni si svolgono con le dichiarazioni programmatiche dei candidati a direttore (tutti catanesi) e con missioni esplorative di funzionari dell' INAF. Nella seduta del 24 marzo 2011 il Consiglio di Amministrazione dell'INAF indica Daniele Spadaro come Direttore dell'Osservatorio catanese. Astronomo associato, nominato il 18 maggio 2011 presenta le dimissioni il 21 maggio 2012. Viene inviato un reggente che dirigerà l'Osservatorio fin tanto che siano espletate le procedure di selezione e nominato il nuovo Direttore.

Dall' 1 dicembre 2012 e con mandato triennale, in base allo Statuto dell' INAF in vigore dal 1 maggio 2011, viene nominata Direttore Grazia Maria Umana: per la prima volta sarà una donna, anche lei formatasi a Catania, a dirigere l'Osservatorio. Con Decreto n. 10 del 17 febbraio 2016 il Presidente dell'INAF le rinnova la nomina fino al 16 febbraio 2019. 
Regolate da appositi atti amministrativi, le procedure di nomina assumono, intanto, un carattere consultivo con ampi margini di pubblicità e selettività. L'art. 18 del Disciplinare di organizzazione e funzionamento, modificato con delibera del Consiglio di Amministrazione n.84/2013 del 19 dicembre 2013, prevede che la selezione per la nomina del Direttore di Struttura sia indetta dal Presidente almeno sei mesi prima della scadenza del Direttore in carica ... abbia un termine non inferiore a venti giorni per la presentazione delle candidature ... avvenga tramite la valutazione comparativa dei curricula dei candidati ... non assimilabile a procedura concorsuale ... una commissione costituita e nominata dal Presidente ... dovrà individuare una rosa di nomi anche attraverso la consultazione del Personale della Struttura interessata ... dovrà redigere una rosa di candidati idonei da inviare al Presidente che procederà alla nomina.

In base al nuovo Statuto dell'INAF approvato dal Consiglio di Amministrazione con delibera 25 maggio 2018 n.42, il nuovo Direttore avrà un incarico di 3 anni e potrà essere confermato una sola volta.

Ho iniziato a scrivere questa disamina all'emanazione del Decreto 42/2018 dell'INAF contenente le procedure di selezione per la nomina del nuovo Direttore dell'Osservatorio Astrofisico di Catania. Espletati tutti i passaggi procedurali previsti è stata nominata Isabella Pagano che sembra voler affermare una nuova caratteristica della Direzione dell'Osservatorio catanese: il rosa. Troverà l'Osservatorio Astrofisico di Catania protagonista in una serie di collaborazioni internazionali che gestiscono missioni spaziali d'avanguardia (GAIA, PLATO, Solar Orbiter, ...) e progetti osservativi da Terra su basi intercontinentali (CTA, SKA, ...), allo sviluppo di buona parte delle quali ha contribuito, essendosi laureata, formata scientificamente e fatto ricerca a Catania. Buon lavoro.

\section{Riferimenti bibliografici}

[1] Blanco C., Consoli S., 2017, L'insegnamento universitario dell'Astronomia ed i tentativi di erigere un Osservatorio a Catania fra '700 e '800, Memorie e Rendiconti dell'Accademia di Scienze Lettere e Belle Arti degli Zelanti e dei Dafnici di Acireale - Serie IV, Vol. II, pp. 213-244.

[2] Reina E., 1839, Elogio del Prof. Francesco Gambino, Atti Acc. Gioenia, Serie II, Vol. 13, pp. 247-250.

[3] Chinnici I., Blanco C., 2013, L'Etna e le stelle: la nascita dell'Osservatorio Astrofisico di Catania, Società Italiana degli Storici della Fisica e dell'Astronomia (SISFA), Atti del XXXIII Convegno Annuale/Proceedings of the $33^{\text {rd }}$ Annual Conference, a cura di L. Fregonese e I. Gambaro, Pavia University Press, pp. 67-83.

[4] Blanco C., 2005, L'Accademia degli Astronomi, in L'Accademia Gioenia. 180 anni di cultura scientifica (1824-2004), a cura di M. Alberghina, Catania, Maimone, pp. 171-177.

[5] Blanco C., 2007, L'innovativa strumentazione del Regio Osservatorio Astrofisico di Catania alla fine dell'Ottocento, in Idee cultura e storia per la Città della Scienza, a cura di M. Alberghina e P. Finocchiaro, Catania, Maimone, pp. 48-55.

[6] Blanco C, Catalano S., Marilli E., Rodonò M., 1983, Twenty years of dedicated photometry of RS CVn at Catania Observatory, IAU Colloq. 71, pp. 387-390. 